\title{
TACTICAL COMBAT CASUALTY CARE: TRANSITIONING BATTLEFIELD LESSONS LEARNED TO OTHER AUSTERE ENVIRONMENTS
}

\section{The Transition to the Committee on Tactical Combat Casualty Care}

\author{
Stephen D. Giebner, MD, MPH \\ Developmental Editor, Committee on Tactical Combat Casualty Care.
}

\begin{abstract}
The original Tactical Combat Casualty Care (TCCC) guidelines were published in a special supplement to Military Medicine in 1996 as the terminal deliverable of a 2-year development project funded by the United States Special Operations Command (USSOCOM). Two years later, the USSOCOM Biomedical Initiatives Steering Committee (BISC) promulgated its Task Statement 5-98, in which it called for the formation of a panel of subject matter experts to update the TCCC guidelines. This article discusses the formation of the Committee on Tactical Combat Casualty Care (CoTCCC) and the changes to the original guidelines that constituted the first update.
\end{abstract}

Keywords: Tactical Combat Casualty Care (TCCC), Committee on Tactical Combat Casualty Care (CoTCCC), combat medic, combat trauma, battlefield trauma, Delphi consensus

\section{Introduction}

Development of Tactical Combat Casualty Care (TCCC) guidelines stemmed from a needs statement issued in 1993 by the commander of the Naval Special Warfare command, calling for a "study on combat casualty care techniques in Special Operations." The United States Special Operations Command (USSOCOM) funded a 2-year study to meet this need, and TCCC was the result. The original TCCC guidelines first appeared in a special supplement to Military Medicine in 1996. ${ }^{1}$ Two years later, the Biomedical Initiatives Steering Committee (BISC) of USSOCOM published Task Statement 5-98, calling for an update to the orignal guidelines. The Naval Operational Medicine Institute (NOMI) responded to that call with a proposal to convene a panel of subject matter experts to draft recommended guideline changes, determine a methodology for consensus development, and propose appropriate financial and administrative support for an iterative update process. The BISC

Corresponding author: Stephen D. Giebner, MD; e-mail: sdgiebner@msn. com.

Presented at the Tactical Combat Casualty Care: Transitioning Battlefield Lessons Learned to Other Austere Environments Preconference to the Seventh World Congress of Mountain \& Wilderness Medicine, Telluride, Colorado, July 30-31, 2016. funded NOMI's proposal as a 2-year development project to begin in 2001.

\section{The concept}

The proposal from NOMI held that a group of subject matter experts could achieve consensus on recommended changes to the guidelines using a modified Delphi technique. This technique was developed in the 1950s and 1960s by the Rand Corporation to address the limitations inherent in achieving consensus by roundtable discussion in committee, namely, arriving at consensus by simple compromise between divergent views instead of in-depth consideration of available information, undue influence of perceived authority or overbearing personality, and unwillingness to abandon opinions already publicly expressed. ${ }^{2}$ The Delphi method replaces committee-like activity with sequential anonymous interrogations, usually via questionnaires, separated by analysis and synthesis of the gathered information. The process is repeated until a predefined consensus is achieved. For quality results, a Delphi study depends upon careful selection of the subject matter experts, a high participation rate among those experts, and avoidance of guiding expert feedback via the formulation of the questionnaires. ${ }^{3}$

Under the NOMI proposal, an iterative anonymous voting procedure (by paper ballot) with discussion and 
modification of the proposed guideline change between rounds of voting would be continued until a two-thirds supermajority was reached. The two-thirds supermajority was chosen as a minimum for adoption because a simple majority could not be viewed as consensus, and a practical limit on the longevity of the procedure was needed because $100 \%$ agreement might never be reached. To assure that perspective, experience, and expertise were the real determinants of the votes, not military rank; each member of the committee, from the most junior medic to the most senior surgeon, was given an equal vote. Furthermore, every member was guaranteed an equal right (and opportunity) to speak and debate.

\section{Membership}

To convene the expert panel, selection of its members was the obvious first step. It was especially important to achieve representation from all services in order to address differences in operational policies and procedures as well as contrasting or conflicting medical practices. It was also important to secure input from the entire spectrum of combat casualty care from the point of injury to definitive care. Participation by Army combat medics, Navy hospital corpsmen, and Air Force pararescuemen - the first responders-was most crucial. Not only did they have vast experience with interventions that had worked and had not worked on the battlefield, they also could provide invaluable advice on what would be acceptable for inclusion in a battlefield first responder's rucksack in terms of need, efficacy, space, and weight. Practical guidance from the end users was considered vital. Medical officers with operational experience were invited for their expertise in managing the medical care projected onto the battlefield and training, equipping, and deploying first responders. Some operational medicine practitioners were selected because of their positions on the staffs of major stakeholder organizations. Trauma surgeons were sought for their knowledge of the presurgical needs and definitive care of combat trauma victims. Medical educators and pharmacists were also invited for their expertise. Some seats were saved for civilian trauma care specialists so the group could accrue the benefits of experience from that community, experience that the military medical system had possibly not provided. Figure 1 depicts the membership of the original Committee on Tactical Combat Casualty Care (CoTCCC).

\section{The process}

Under the development project, the committee met 3 times over 2 years. It became obvious during the first
The Committee on Tactical Combat Casualty Care: 2002

Chairman - CAPT Stephen Giebner

\begin{tabular}{lll} 
COL Robert Allen & LTC Stephen Flaherty & Dr. Norman McSwain \\
COL Frank Anders & CDR Scott Flinn & SFC Robert Miller \\
CPT Steve Anderson & MAJ John Gandy & MAJ Kevin O'Connor \\
COL James Bagian & CAPT Larry Garsha & CAPT Edward Otten \\
COL Ron Bellamy & COL John Holcomb & LTC Tyler Putnam \\
1LT Bart Bullock & Dr. David Hoyt & CDR Peter Rhee \\
CAPT Frank Butler & LTC Donald Jenkins & CAPT Larry Roberts \\
Dr. Howard Champion & COL Jay Johannigman & CDR Jeff Timby \\
TSGT George Cum & MSG John Kennedy & HMCM Gary Welt \\
CAPT Roger Edwards & CPT Robert Mabry & \\
\multicolumn{1}{c}{ Executive Assistants: LT David Anderson, Ms. Shannon Addison }
\end{tabular}

Figure 1. The membership of the original CoTCCC.

meeting that anonymous iterative voting would not be needed. The group was consistently able to reach consensus after simple open discussion and debate. The one-vote-per-member and equal-right-to-speak approach were readily adopted and broadly respected. This approach did much to reduce the possible influence of domineering personalities, unwillingness to openly disagree with authority figures, and the vocality of a passionate minority masking the true opinion of a quiet majority - the aforementioned problems inherent in committee activity. The anonymity provided in a straight Delphi approach would have provided similar effect ${ }^{4}$ but would have consumed a great deal more time. The rules and procedures established in those first 3 meetings have continued to the present and are ingrained as standard operating procedure.

\section{The result: TCCC 2003}

The 2003 TCCC guidelines, the first update rendered by the committee, were published with extensive explanatory text in the military version of the revised fifth edition of the Prehospital Trauma Life Support textbook..$^{5}$ The new guidelines were also presented in contrast with the original guidelines by this author in the Journal of Special Operations Medicine. ${ }^{6}$ A comparison of the 1996 guidelines (Figures 2, 4, and 6) and the 2003 guidelines (Figures 3, 5, and 7), showing the changes made in each phase of care, is presented again here. Changes are presented in italics.

\section{CARE UNDER FIRE}

Care Under Fire is the phase of care on the battlefield during which the casualty and the medic are under effective hostile fire. The 1996 Care Under Fire guidelines are shown in Figure 2, and the 2003 version in Figure 3.

The first point of difference-having the wounded continue as combatants when possible-was especially 


\section{Care Under Fire - 1996}

1. Return fire as directed or required

2. Try to keep yourself from getting shot

3. Try to keep the casualty from sustaining additional wounds

4. Airway management is generally best deferred until the Tactical Field Care phase

5. Stop any life-threatening external hemorrhage with a tourniquet

6. Take the casualty with you when you leave

Figure 2. The 1996 TCCC guidelines for Care Under Fire.

important to special operations forces whose maneuvering elements are small in number. In these small elements, having as many guns as possible in action was considered vitally important. Any casualty whose wounds were not incapacitating would be expected not only to continue the firefight, but also to finish the mission.

The second change was a recommendation to use the HemCon Bandage (Hemcon Medical Technologies Inc, Portland, OR) to control nonextermity hemorrhage. HemCon is a chitosan dressing that demonstrated efficacy in laboratory trials and could be readily applied under combat conditions. The committee recommended that all combatants carry and be able to apply both a limb tourniquet and a hemostatic dressing to provide a robust capability for early hemorrhage control on the battlefield.

The third change in Care Under Fire was a recommendation for caregivers to explain to the casualty the care they were giving and to offer encouragement because these actions were known to be beneficial to trauma patients.

The direction to bring along the casualties when the unit departed was dropped from the guidelines because it was a tactical decision, not a medical one.

\section{Care Under Fire - 2003}

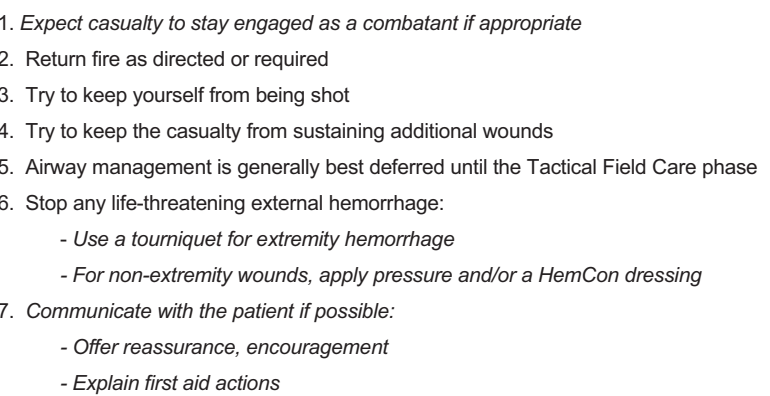

Figure 3. The 2003 TCCC guidelines for Care Under Fire. Represents the first update rendered by the CoTCCC. Changes are presented in italics.

\section{$\underline{\text { Tactical Field Care - } 1996}$}

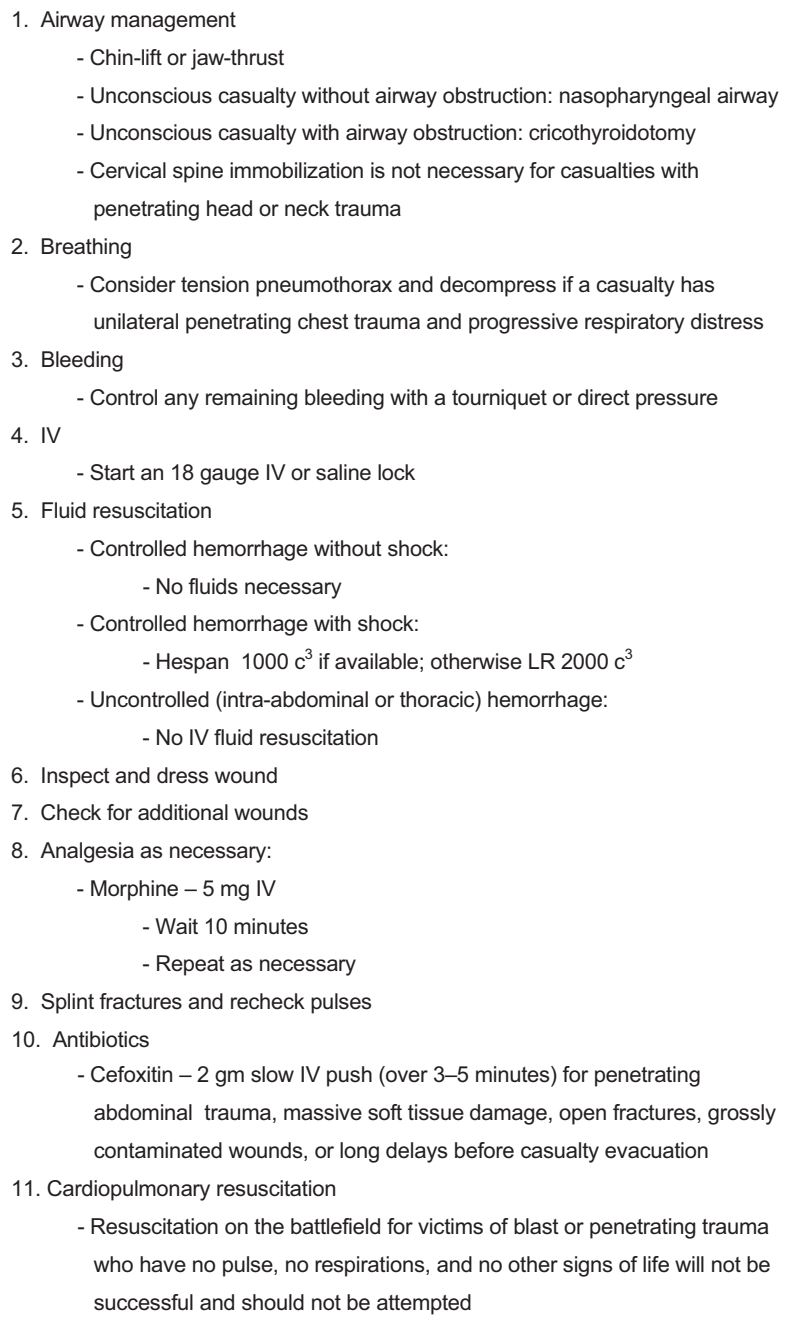

Figure 4. The 1996 TCCC guidelines for Tactical Field Care (TFC).

\section{TACTICAL FIELD CARE}

Tactical Field Care (TFC) is any phase of care on the battlefield during which the casualty and the medic are not under effective hostile fire, but the casualty is not yet being evacuated. This circumstance may occur before or after engagement with the enemy. The 1996 TFC guidelines and the 2003 update for that phase are depicted in Figures 4 and 5, respectively. The first change in TFC was the addition of a tactical safety measure as the first step: All weapons should be collected from any casualty with altered mental status to prevent their inadvertent or inappropriate employment. The committee modified the airway management guideline to give medics a progressive scheme of intervention, from simple to more invasive. Chin lift or jaw thrust to initially open the airway followed by insertion of a nasopharyngeal airway and then placing 


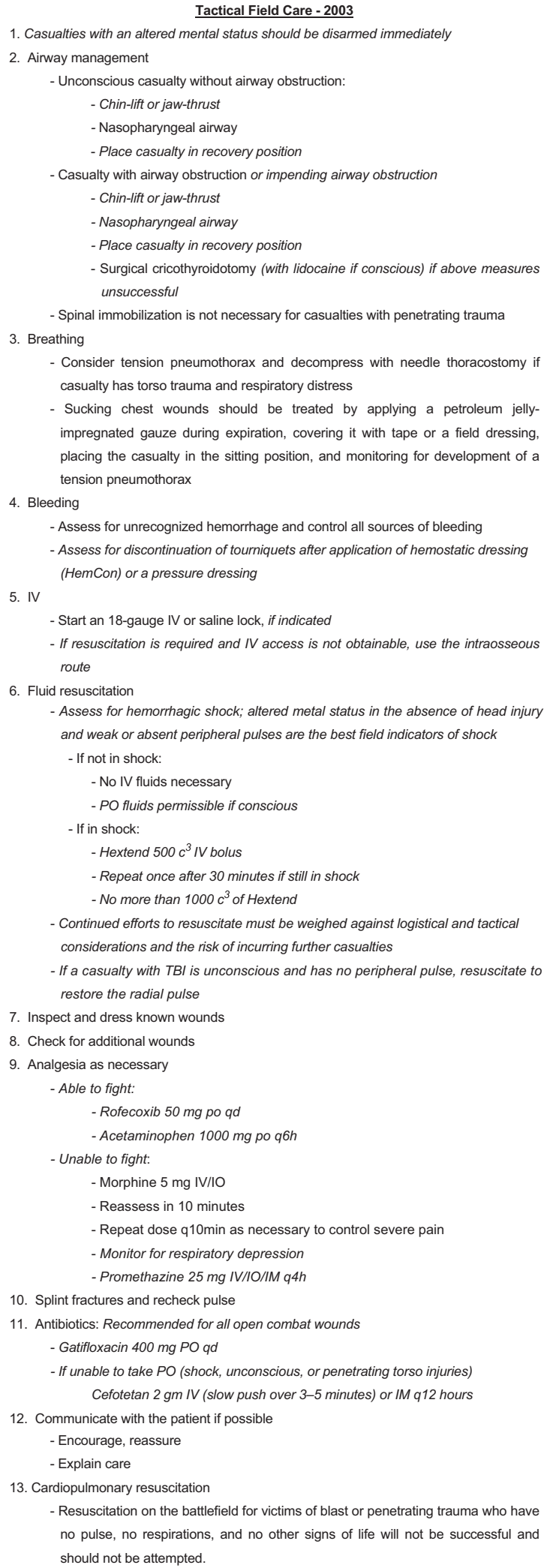

Figure 5. The 2003 TCCC guidelines for Tactical Field Care. Represents the first update rendered by the CoTCCC. Changes are presented in italics. the casualty in the recovery position was recommended for unconscious casualties with no airway obstruction. For casualties (conscious or not) with overt or impending airway obstruction, the medic was directed to proceed directly to surgical crycothyroidotomy if the simple measures just described proved insufficient. Endotracheal intubation was not recommended as a battlefield intervention by combat medics because crycothyroidotomy was throught to provide a higher probability of success.

The breathing guideline was expanded to include battlefield management of sucking chest wounds. The committee recommended construction of chest seals using petroleum jelly-impregnated gauze over these wounds, with a reminder to monitor for tension pneumothorax thereafter.

Advice to convert limb tourniquets to HemCon dressings or pressure dressings when possible was added to the section on hemorrhage control. This was deemed a necessary subsequence to the application of a $\operatorname{limb}$ tourniquet to minimize the risks of ischemic and compressive injury.

Acknowledging that some casualties would not require intravenous fluids or medications, the committee appended the phrase "if indicated" to the recommendation for intravenous access. The committee also pointed out that the intraosseous route was an acceptable alternative to the intravenous. Contemporary intraosseous devices were safe, could be quickly applied, and could be successfullly employed when intravenous access proved difficult. Furthermore, the recommended analgesics and antibiotics could be given intraosseously. Of the available devices, the committee considered the FAST-1 sternal intraosseous device (Pyng Medical Corporation, Richmond, British Columbia, Canada) best suited for tactical environments.

The committee made extensive modifications to the guideline for fluid resuscitation. A practical clinical definition of hemorrhagic shock was provided by inserting 2 clinical indicators the committee believed could be reliably assessed on the battlefield: altered metal status in the absence of head injury and weak or absent peripheral pulses.

The new recommendation for oral fluids for casualties with penetrating trauma, a normal state of consciousness, and the ability to swallow was made on the basis of 2 important observations. First, casualties were markedly dehydrated upon arrival at forward-deployed treatment facilities. Second, the actual risk of emesis and aspiration in trauma surgery centers among alert patients with penetrating trauma was remarkably low.

The Hextend formulation of hetastarch (BioTime, Inc., Alameda, CA) was the committee's first choice of 
Casualty Evacuation (CASEVAC) Care -1996

1. Airway management

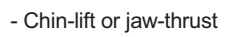

- Unconscious casualty without airway obstruction:

- Nasopharyngeal airway, endotracheal intubation, Combitube or laryngeal mask airway

- Unconscious casualty with airway obstruction:

- Cricothyroidotomy if endotracheal intubation and/or other airway devices are unsuccessful

2. Breathing

- Consider tension pneumothorax and decompress with needle thoracostomy if a casualty has unilateral penetrating chest trauma and progressive respiratory distress

- Consider chest tube insertion if a suspected tension pneumothorax is not relieved by needle thoracostomy

- Oxygen

3. Bleeding

- Consider removing tourniquets and using direct pressure to control bleeding if possible

4. IV

- Start an 18 gauge IV or heparin lock if not already done

5. Fluid resuscitation

- No hemorrhage or controlled hemorrhage without shock:

- Lactated Ringers at $250 \mathrm{cc} / \mathrm{h}$

- Controlled hemorrhage with shock:

- Hespan 1000 cc/h initially if available; otherwise LR 2000 cc

- Uncontrolled (intra-abdominal or thoracic) hemorrhage:

- No IV fluid resuscitation

- Head wound patient:

- Hespan at minimal flow to maintain infusion unless there is concurrent controlled hemorrhagic shock

6. Monitoring

- Institute electronic monitoring of heart rate, blood pressure, and

hemoglobin oxygen saturation

7. Inspect and dress wound if not already done

8. Check for additional wounds

9. Analgesia as necessary:

- Morphine - 5 mg IV

- Wait 10 minutes

- Repeat as necessary

10. Splint fractures and recheck pulses if not already done

11. Antibiotics (if not already given):

- Cefoxitin - 2 gm slow IV push (over 3-5 minutes) for penetrating

abdominal trauma, massive soft tissue damage, open fractures, grossly

contaminated wounds, or long delays before casualty evacuation

Figure 6. The 1996 TCCC guidelines for Casualty Evacuation Eare (CASEVAC).

resuscitation fluids for use on the battlefield. Although it had not been widely used as a prehospital resuscitation fluid, a prolonged physiologic effect using minimal volumes had been reported in clinical settings. A protective influence against multiple organ injury after hepatoenteric ischemia-reperfusion had also been reported. The 2003 update recommended that all casualties in shock be given a $500 \mathrm{~mL}$ rapid intravenous bolus of Hextend, and if no improvement was noted in 30 minutes, the bolus was to be repeated once. Resuscitation beyond that point would be driven by clinical judgment, supplies on hand, and the tactical situation. This simple regimen offered important advantages: 1) titration to the desired clinical effect would conserve both fluids and time; 2) administration of fluids directed by a monitored response could possibly avoid an excessive rise in blood pressure leading to rebleeding from previously clotted sites; and 3) tailoring fluid therapy to the casualty's clinical response allowed for a single approach to resuscitation in both controlled and uncontrolled hemorrhage.

The last change to the fluid resuscitation guideline was a resuscitation goal specifically for casualties with traumatic brain injury (TBI) and shock. Because hypotension was associated with increased mortality in the presence of TBI, resuscitation with intravenous or intraosseous fluids to achieve a palpable radial pulse, corresponding to a systolic blood pressure of at least $80 \mathrm{~mm} \mathrm{Hg}$, was recommended for these casualties.

In the 1996 guidelines, analgesia was limited to a simple regimen of intravenous morphine sulfate. The committee expanded this section to include a per oral regimen for mild to moderate pain for those casualties who could continue as combatants. Acetaminophen and rofecoxib, given together, were expected to provide appreciable pain relief without altering the sensorium. Rofecoxib (Vioxx [Merck \& Co., Inc., Kenilworth, NJ]), a cyclo-oxygenase-2 inhibitor, caused no platelet dysfunction, had a milder side effects profile than other cox2 inhibitors, and carried no contraindication in sulfasensitive individuals. The committee also added a recommendation for promethazine to combat the nausea and vomiting often associated with the use of morphine.

The antibiotic regimen was changed significantly. First, the committee recommended antibiotics for any significant break in the skin occurring on the battlefield and not just for extensive injuries or long delays, as in the original guidelines. This change was intended to combat the increased risk of morbidity and mortality associated with wound infections. A per oral regimen was added here, too, with gatifloxacin, a fourthgeneration flouroquinolone, emerging as the agent of choice. Gatifloxacin's desirable characteristics included a very broad spectrum of activity, including Vibrio and Aeromonas species of interest in aquatic operations; once-daily dosing allowing for minimal weight and volume in the medic's rucksack; a mild side effects profile; environmental stablity; and competitive pricing. For casualties requiring intravenous antibiotics, the committee recommended cefotetan for similar considerations. 


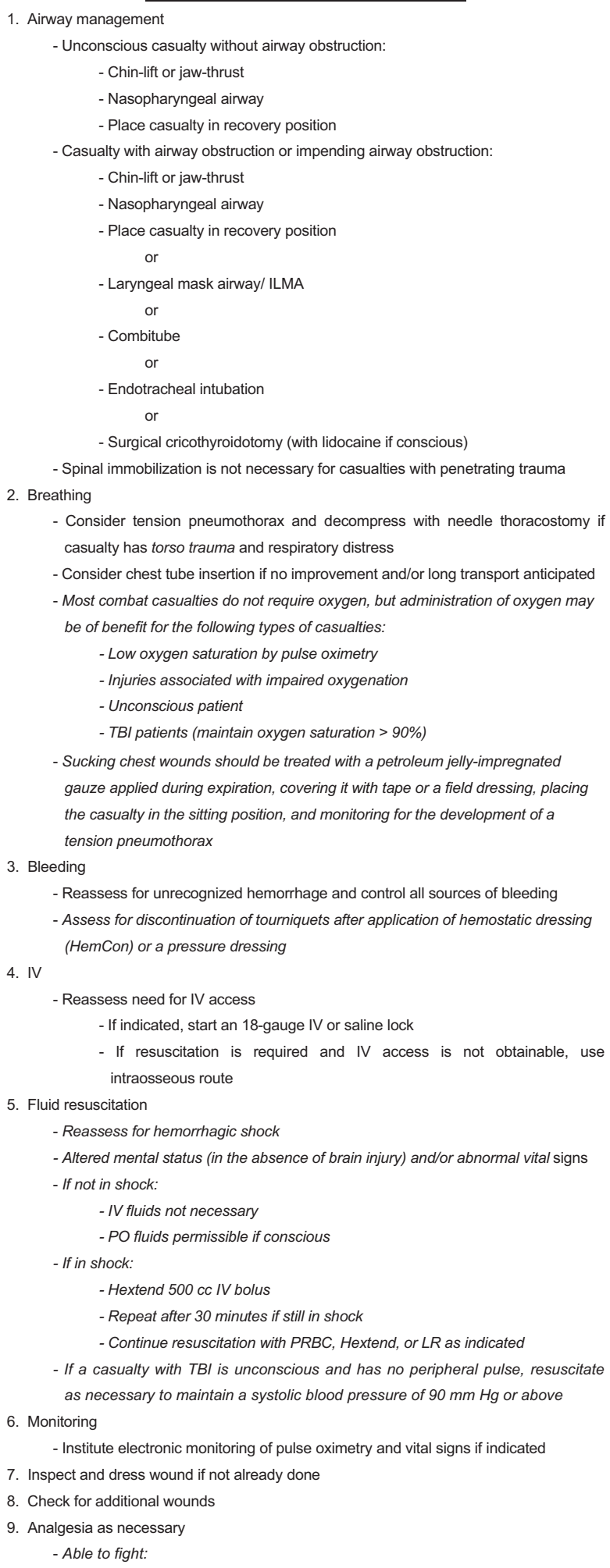

- Reassess for unrecognized hemorrhage and control all sources of bleeding

- Assess for discontinuation of tourniquets after application of hemostatic dressing (HemCon) or a pressure dressing

Figure 7. The 2003 TCCC guidelines for Casualty Evacuation Care (CASEVAC). Represents the first update rendered by the CoTCCC. Changes are presented in italics. ILMA = intubating laryngeal mask airway.

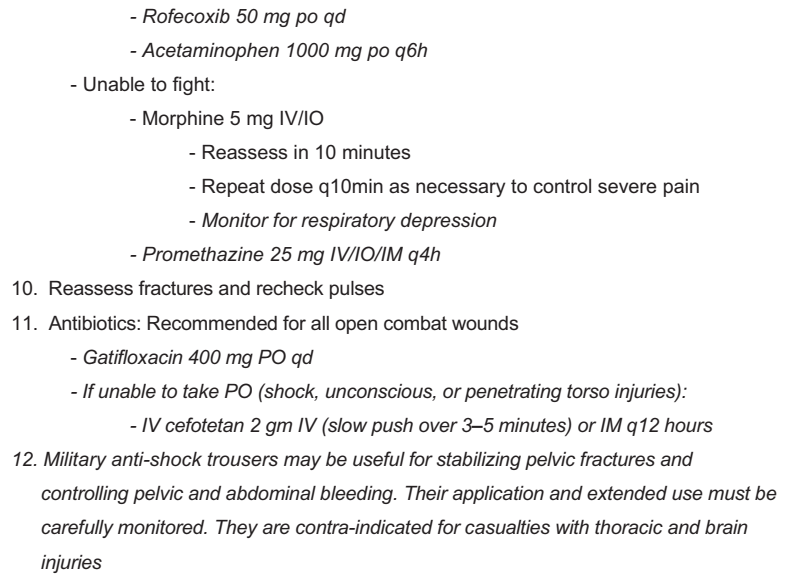

Figure 7. Continued.

\section{CASUALTY EVACUATION CARE}

Casualty Evacuation (CASEVAC) Care occurs while the casualty is being transported off the battlefield to a medical treatment facility. The 1996 and 2003 guidelines for this phase are presented in Figures 6 and 7. More advanced care may be possible during CASEVAC than in TFC because additional medical equipment and personnel should be available on the extraction platform. CASEVAC guidelines differ from TFC guidelines only where more advanced options for trauma care are suggested. Preplanning for CASEVAC optimally includes more airway control alternatives, oxygen as needed, options for continued fluid resuscitation, and more advanced electronic monitoring. Beyond those changes in CASEVAC that reflect the changes the committee made in TFC, a final recommendation for military anti-shock trousers was added for the treatment of pelvic fractures in CASEVAC to stabilize pelvic fractures and to help control pelvic and abdominal bleeding.

\section{Transition to Permanence}

As required in the task statement, at the end of the project the committee made recommendations for continually updating the TCCC guidelines. These were as follows:

- The CoTCCC should be made into a permanent, standing body.

- The CoTCCC should monitor advances in medicine and continuously update the TCCC guidelines.

- The CoTCCC should be provided dedicated funding adequate to its mission.

- The position of chairman should be made permanent.

The USSOCOM BISC concurred with these recommendations. As a result of tireless campaigning by the 
commanding officer of NOMI, Captain Doug Freer, the Navy Bureau of Medicine and Surgery agreed to provide line-item funding for continuation of the CoTCCC. A civilian physician position was created at NOMI for the chairman.

The CoTCCC has continued its work for 15 years. Today, it is the prehospital arm of the Joint Trauma System. The Joint Trauma System is administratively supported by and a tenant command aboard the United States Army Institute of Surgical Research, Brooks Army Medical Center, San Antonio, Texas. Interested readers can find contemporary TCCC guidelines and teaching materials released by the CoTCCC at http:// www.naemt.org/education/TCCC/tccc.aspx or http:// www.specialoperationsmedicine.org/Pages/tccc.aspx.

Acknowledgments: The Naval Operational Medical Institute that facilitated the original development project, and the project's sponsor, the United States Special Operations Command Biomedical Initiatives Steering Committee, deserve special thanks for making the creation of the CoTCCC and its transition to permanence possible. The author would like to express a special appreciation for the hard work of the members, advisors, and support staff of the CoTCCC and for the enthusiasm with which they embrace their work. No acknowledgment would be complete without recognizing the military first responders who put their lives on the line to aid their wounded teammates. The committee exists to serve them and their patients.

Financial/Material Support: None.

Disclosures: None.

\section{References}

1. Butler F, Hagmann J, Butler E. Tactical combat casualty care in special operations. Mil Med. 1996;161:3-16.

2. Helmer-Hirschberg, O. Analysis of the future: the Delphi method. The Rand Corporation Web site. Available at: http://www.rand.org/pubs/papers/P3558.html. March, 1967. Accessed September 23, 2016.

3. Hsu CC, Sandford BA. The Delphi technique: making sense of consensus. Pract Assess Res Eval. 2007;12. Available at: http://pareonline.net/pdf/v12n10.pdf. Accessed September 23, 2016.

4. Goodman CM. The Delphi technique: a critique. J Adv Nurs. 1987;12:729-734.

5. Adkisson GH, Beard ML, Butler FK, et al. Military medicine. In: McSwain N, ed. Prehospital Trauma Life Support. Revised 5th ed. St Louis, MO: Mosby; 2003:374408.

6. Giebner SD. Tactical Combat Casualty Care - 2003. J Spec Oper Med. 2003;3. Available at: http://www.socom.mil/ JSOMDocs/Forms/JSOM.aspx. Accessed September 23,2016. 